\title{
Astrometry of Pluto and Saturn with the CCD meridian instruments of Bordeaux and Valinhos ${ }^{\star}$
}

\author{
M. Rapaport ${ }^{1,2}$, R. Teixeira ${ }^{2,1}$, J. F. Le Campion ${ }^{1,2}$, C. Ducourant ${ }^{1}$, \\ J. I. B. Camargo ${ }^{2}$, and P. Benevides-Soares ${ }^{2}$
}

\author{
1 Observatoire de Bordeaux, BP 89, 33270 Floirac, France \\ 2 Departamento de Astronomia, Universidade de São Paulo, Av. Miguel Stéfano 4200, \\ 04301-904 São Paulo, Brazil
}

Received 3 October 2001 / Accepted 21 November 2001

\begin{abstract}
The accuracy of CCD meridian circles observations performed at the Bordeaux and Valinhos Observatories confirms the improvement represented by the DE405 ephemeris when compared to DE403 for Saturn and Pluto. In this work, we also use published positions obtained with the Flagstaff CCD meridian circle. We compare the DE403 and DE405 ephemerides and show that for Pluto and Saturn, large differences appear (100 mas and 60 mas respectively). The CCD meridian observations of Pluto prove that the DE405 ephemeris better represents the observations than does DE403. In the case of Saturn, the comparison has to be done on the satellite positions since the planet is too bright to be observed with the meridian circles. First we compare the two current major theories (Vienne \& Duriez 1995; Harper \& Taylor 1993, hereafter VD95 and HT93) of the eight first satellites of Saturn. We show in the case of Iapetus that there is an oscillation in the residuals coming from HT93 and that VD95 better represents the observations. We conclude on the necessity to continue ground-based observations of the outer planets and of their satellites to discriminate between proposed theories and ephemerides.
\end{abstract}

Key words. astrometry - ephemerides - planets and satellites: general

\section{Introduction}

Acquisition of precise astrometric positions of the outer planets and their satellites is essential for the improvement and maintenance of their ephemerides. This improvement is important for the preparation of space missions, for the prediction of occultations of stars by planets or satellites and their visibility zone. The outer planets are too far to be observed with the very accurate methods used to observe the closer planets (Viking range observations, radar, interferometry, lunar laser ranging data). It is therefore necessary to observe outer planets such as Saturn and Pluto with ground-based instruments.

In the years following the publication of DE200 ephemerides (Standish 1990) the comparison of observed and calculated positions for the outer planets exhibited significant systematic differences. In the case of Pluto, dif-

\footnotetext{
Send offprint requests to: M. Rapaport, e-mail: rapaport@observ.u-bordeaux.fr

* Table 1 is only available in electronic form at the CDS via anonymous ftp to cdsarc.u-strasbg.fr (130.79.128.5) or via

http://cdsweb.u-strasbg.fr/cgi-bin/qcat?J/A+A/383/1054
}

ferences reached over 3 arcsec and were increasing very rapidly with time. Systematic differences existed also for Uranus and Neptune. In the case of Pluto, the degradation of the ephemerides could be explained by the small fraction, $1 / 4$ of the orbital period, covered by astrometric observations. New versions of the ephemerides were then published (DE403, DE405, DE406) correcting most of these systematic effects. In parallel, new astrometric observations of the outer planets and of their satellites have been performed with the CCD meridian circles of Flagstaff (Stone 2000), Valinhos and Bordeaux (Viateau et al. 1999).

In this work we compare, for the outer planets, the observations from Bordeaux, Valinhos and Flagstaff made over the period 1995-2001 with the recent ephemerides DE403 and DE405 from JPL.

\section{Comparison of DE403 and DE405 ephemerides for the outer planets}

We present in Fig. 1 the differences for Saturn, Uranus, Neptune and Pluto between the DE403 and DE405 ephemerides for the period 1985-2010. For Uranus and 

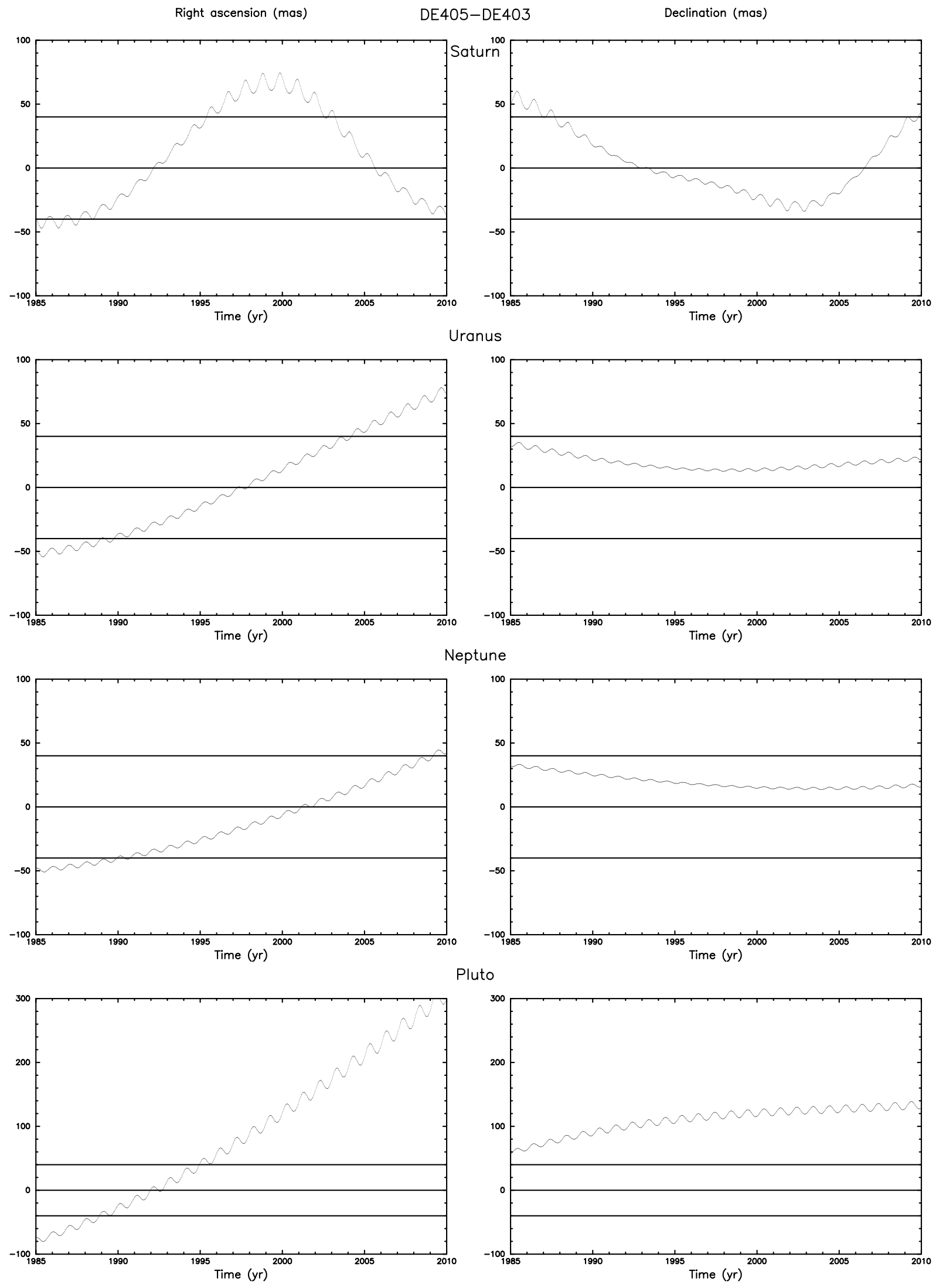

Fig. 1. Comparison of DE403 and DE405 ephemerides for the outer planets for the period 1985-2010. 

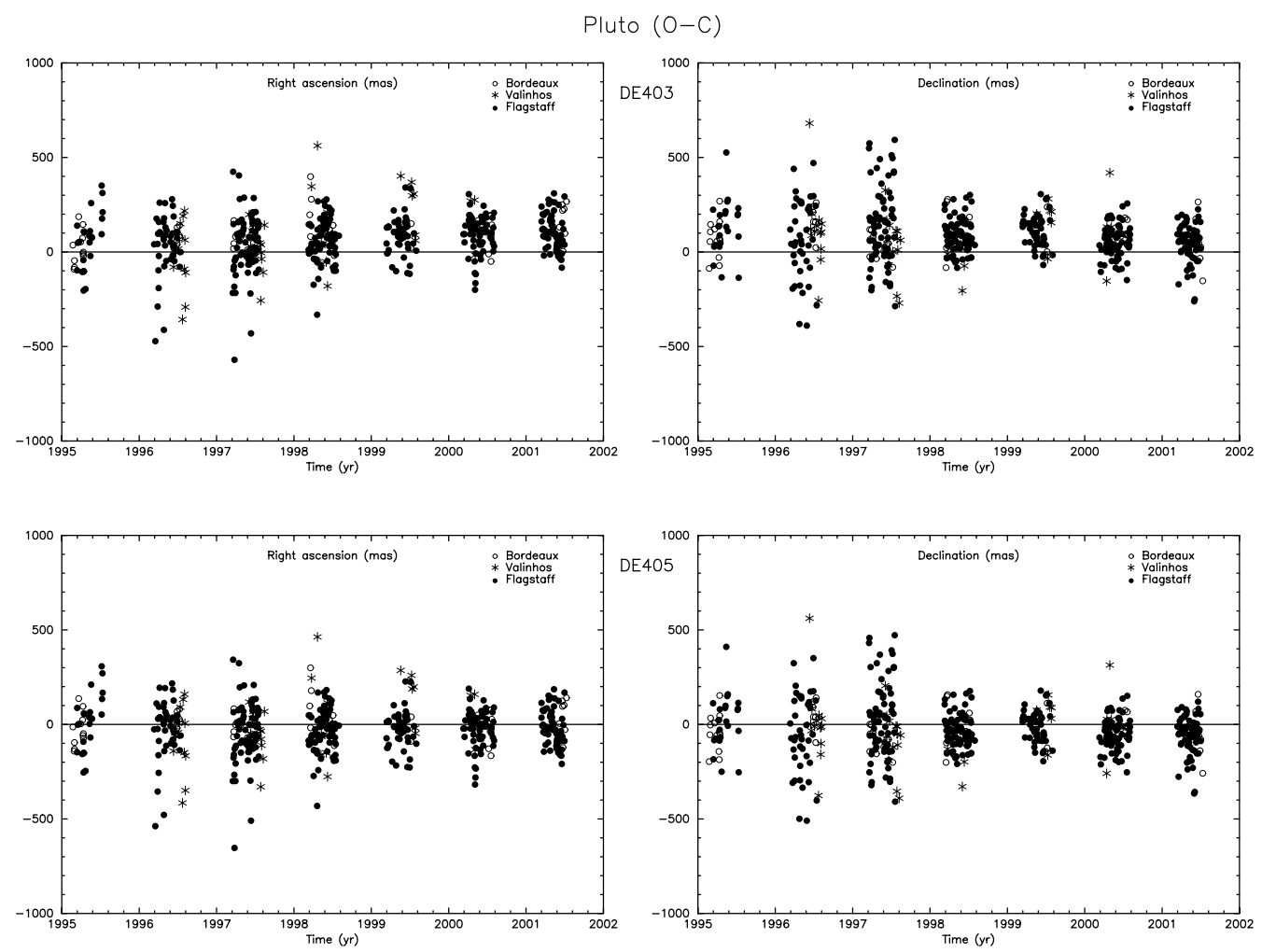

Fig. 2. Comparison of meridian observations (Bordeaux, Valinhos, Flagstaff) of Pluto and DE403 and DE405 ephemerides.

Neptune, the differences between positions given by the two ephemerides (DE403 and DE405) for the period 1995-2002 are small, less than 20 mas. In the case of Saturn and Pluto the situation is different since the differences between the two ephemerides reach 100 mas for Pluto and 60 mas for Saturn.

Taking into consideration the precision of CCD meridian observations, the differences between DE403 and DE405 for Saturn and Pluto can be investigated with our instruments.

\section{Data}

We have acquired a very good set of CCD meridian observations of Pluto and of the major satellites of Saturn: Dione, Rhea, Titan, Hyperion and Iapetus from 1995 to 2001 at the Bordeaux and Valinhos Observatories. The Bordeaux and Valinhos observatories are located at the coordinates: Bordeaux $\left(L_{\mathrm{w}}=00^{\circ} 31.7^{\prime}, \Phi=+44^{\circ} 50.1^{\prime}\right.$, elevation $=73 \mathrm{~m})$, Valinhos $\left(L_{\mathrm{w}}=46^{\circ} 58.0^{\prime}, \Phi=\right.$ $-23^{\circ} 00.1^{\prime}$, elevation $\left.=850 \mathrm{~m}\right)$. These instruments are very similar and give a mean accuracy for one observation ranging from 70 mas to 150 mas, depending on the magnitude. The characteristics of the instruments can be found in Viateau et al. (1999).

We give in Table 1 the observed topocentric mean positions expressed in the ICRF system.

\section{Comparison of meridian observations and ephemerides DE403 and DE405 for Pluto}

The differences between observed and calculated positions of Pluto using respectively DE403 and DE405 are shown
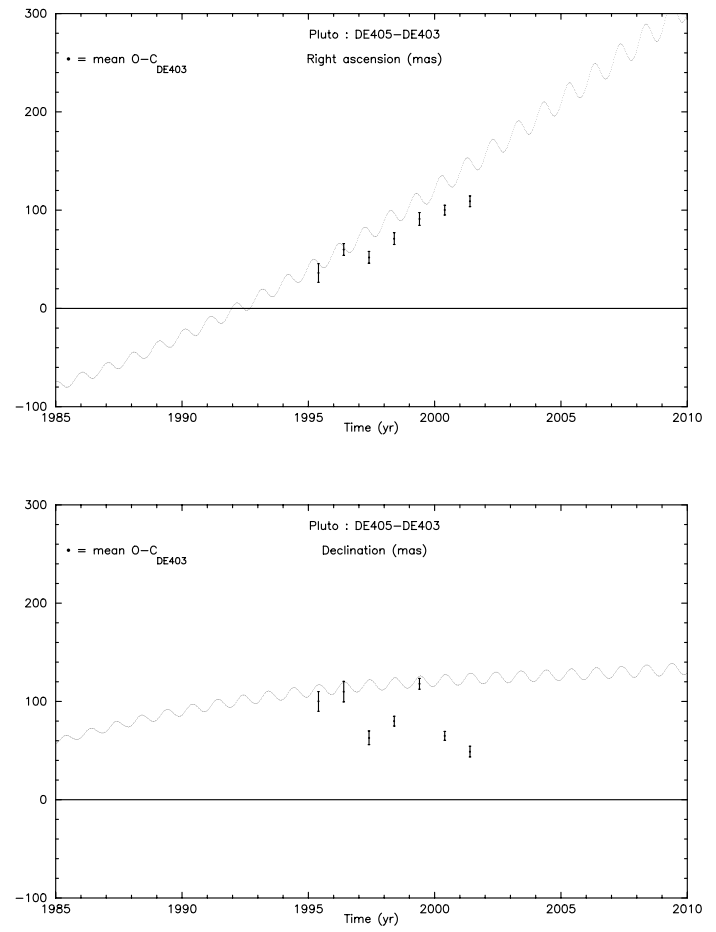

Fig. 3. Comparison of DE403 and DE405 ephemerides for Pluto. Points represent the mean differences between meridian observations and DE403 at each opposition.

in Fig. 2. At first sight one can notice a stream in the behaviour of the $\mathrm{O}-\mathrm{C}_{\mathrm{DE} 403}$ in right ascension and in declination. No effect of this type can be noticed in the $\mathrm{O}-\mathrm{C}_{\mathrm{DE} 405}$. 
This result suggests that the DE405 ephemeris better represents the observations of Pluto than DE403.

To evaluate how much better DE405 represents the observations, we give in Fig. 3 a zoom of Fig. 1 for Pluto. We add in this figure for the mean epoch of each opposition, the mean $\mathrm{O}-\mathrm{C}_{\mathrm{DE} 403}$.

In right ascension there is a good agreement between O- $\mathrm{C}_{\mathrm{DE} 403}$ values and the curves DE405 - DE403 (that is to say between observations and DE405) although one can notice a slight offset (about 40 mas).

In declination there is an improvement of about 60 mas when DE405 is used. Large differences still remain, perhaps due to observations, but a large part of the systematic effect disappears in the $\mathrm{O}-\mathrm{C}_{\mathrm{DE} 405}$ comparison.

In the case of Pluto it is clear that the CCD meridian observations performed in recent years confirm the improvements in the ephemerides DE405 with respect to DE403. However the results obtained (Fig. 3), especially in the case of right ascension, show that it is necessary to continue the astrometric observations of Pluto to understand the nature of the offsets between the observations and DE405.

\section{Comparison of meridian observations and ephemerides DE403 and DE405 for Saturn}

\subsection{Presentation}

Figure 1 shows that for Saturn in the period 1995-2003, the right ascensions determined respectively with DE403 and DE405 differ by about 50 mas, reaching 60 mas at certain moments. It is reasonable to try to investigate these discrepancies by comparing each ephemeris with the meridian observations. The differences in declinations never exceed 40 mas and therefore will remain difficult to detect with meridian observations.

The situation for Saturn differs from the situation of Pluto because the planet is too big to be directly observable. The satellites of Saturn however can be used and the position of Saturn deduced from the observed position of its satellites (Morrison \& Evans 1998). The position of Saturn is obtained by subtracting the calculated differential coordinates of the observed satellite from the observed satellite position. Therefore the resulting position of Saturn observed depends on the theory used for the satellites and we thus have first to compare the different available theories for satellites.

\subsection{Two theories (VD95 and HT93) for the eight first satellites of Saturn}

We will consider here two independent theories of the eight first satellites of Saturn (Vienne \& Duriez 1995; Harper \& Taylor 1993). These theories led to the construction of new ephemerides (hereafter VD95 and HT93). In both cases, the authors take into account all the observations done during more than a century to adjust the integration constants.
We present in Fig. 4 the differences between the geocentric positions for the seven first satellites of Saturn, calculated with VD95 and HT93 for the time interval: 1900-2010 (Hyperion was not considered in HT93).

In this figure one can see that for all satellites, except Mimas and Iapetus, differences between VD95 and HT93 remain much lower than 100 mas. In the case of Mimas and Iapetus, they are much greater than 100 mas and it is then clear that we cannot use these satellites to derive the position of Saturn. However we can use the meridian observations of these two satellites to discriminate their two ephemerides (VD95, HT93). Because of its proximity to Saturn it is not possible to observe Mimas with the CCD meridian circles. Iapetus is further from the planet and we have a large number of observations of this satellite made with the meridian circles.

\subsection{Comparison for lapetus of VD95 and HT93 theories}

Morrison \& Evans (1998) already noticed that residuals of individual observations of Iapetus with respect to HT93 show sinusoidal oscillations with an amplitude reaching 100 mas and a period very close to 79.92 days, corresponding to the synodic period of Iapetus. They suggested that this oscillation could come from a deficiency of HT93 and also suggested a comparison of HT93 and VD95. We give in Fig. 5 the difference for Iapetus of the geocentric calculated coordinates $\mathrm{C}_{\mathrm{VD} 95}-\mathrm{C}_{\mathrm{HT} 93}$ for the period 1985-2010. It is clear in this figure that there is an oscillation with a period close to 80 days, already mentioned by Morrison \& Evans (1998). The spectral analysis of these differences is given in Fig. 6. This figure confirms that the period of the oscillation is effectively 80 days. We will now try to discriminate the theories of VD95 and HT93 by comparing the calculated positions of Iapetus with the meridian observations.

\subsection{Comparison between observations and theories of lapetus}

Here we want to compare the CCD meridian observations of Iapetus with the positions issued from the two theories of satellites VD95 and HT93. The calculated position of Iapetus is obtained using DE405 for Saturn's position and VD95 or HT93 for the differential coordinates of Iapetus with respect to Saturn. We could have used DE403 instead of DE405 for the position of Saturn since the differences between DE403 and DE405 for Saturn range around 50 mas i.e. much less than the expected differences for Iapetus between observations and theories (200 mas).

We present in Fig. 7 the $\mathrm{O}-\mathrm{C}_{\mathrm{DE} 405+\mathrm{VD} 95}$ and the $\mathrm{O}-\mathrm{C}_{\mathrm{DE} 405+\mathrm{HT} 93}$. We clearly see a sinusoidal signal in the $\mathrm{O}-\mathrm{C}_{\mathrm{DE} 405+\mathrm{HT} 93}$ that is absent from the $\mathrm{O}-\mathrm{C}_{\mathrm{DE} 405+\mathrm{VD} 95}$. This strongly suggests that for Iapetus, the VD95 theory better represents the observations than the HT93 theory. 

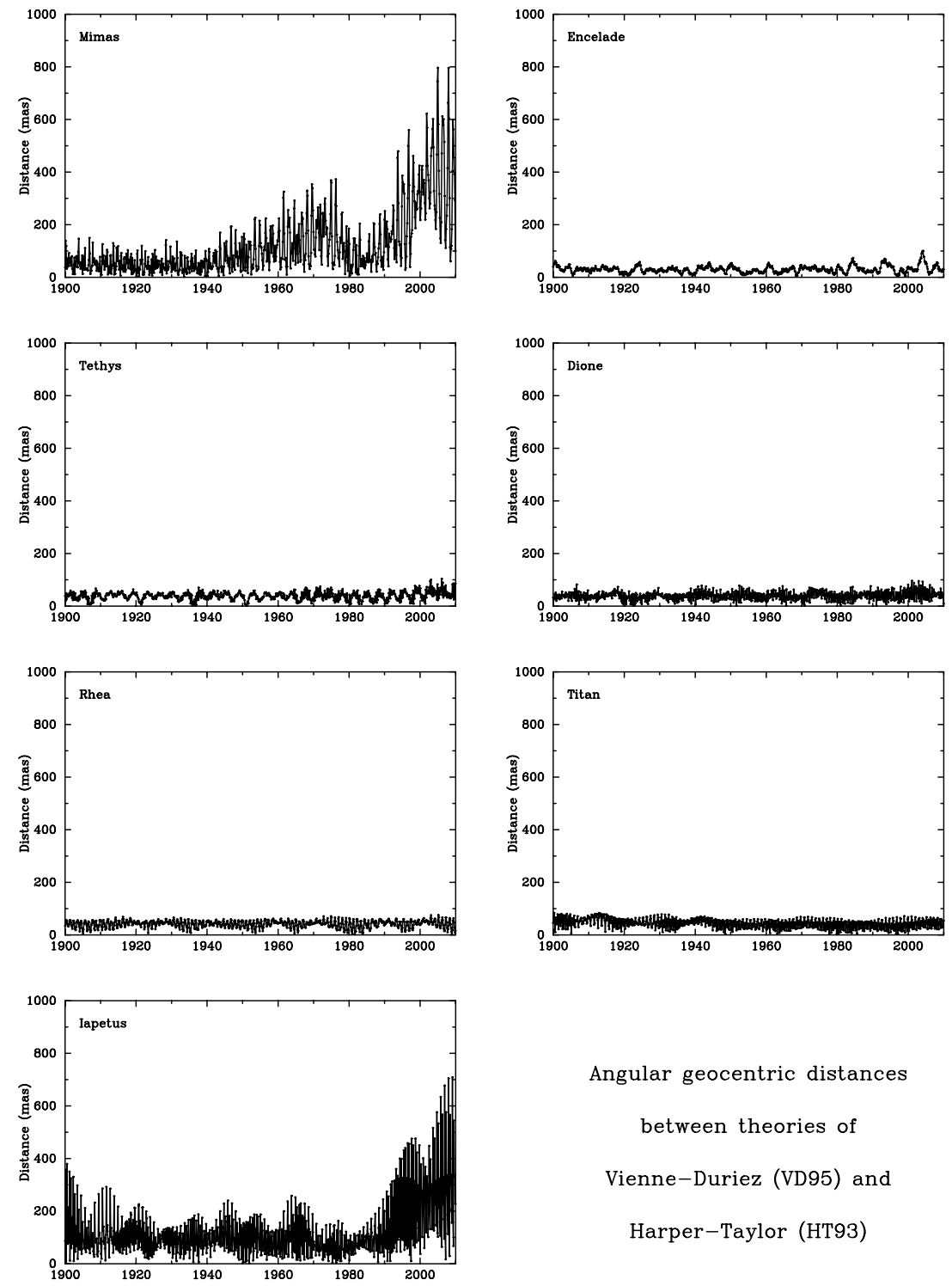

Fig. 4. Angular geocentric distances between VD95 and HT93 theories for the first seven satellites of Saturn.

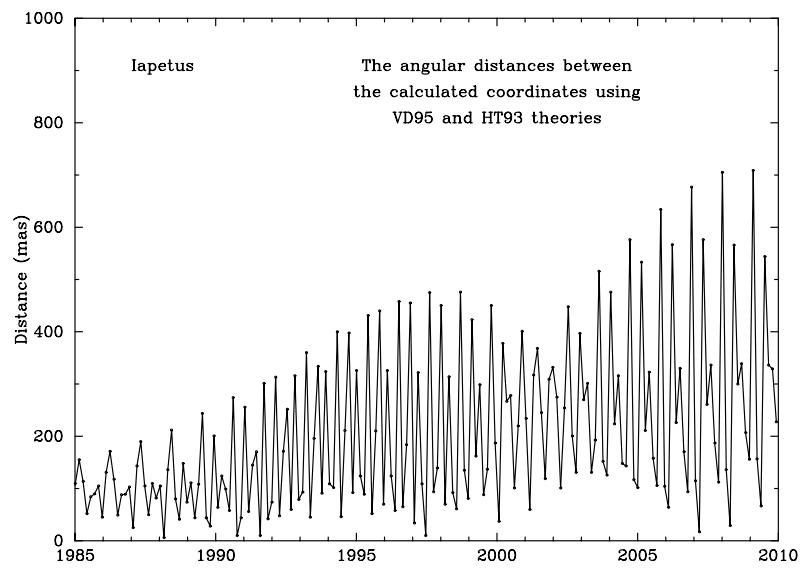

Fig. 5. The angular distances between the calculated coordinates using VD95 and HT93.

A confirmation of this result is obtained by the analysis of the observations of Iapetus made between 1985 and 1997 by the Carlsberg Automatic meridian circle (Helmer \& Morrison 1985) and the photometric meridian of Bordeaux (Réquième \& Mazurier 1986). In Fig. 8 we give the $\mathrm{O}-\mathrm{C}$ between these observations and the two theories (HT93 and VD95) as a function of time modulo 80 days. The sinusoidal signal appears clearly in right ascension when the HT93 theory is used. In declination no clear conclusion can be drawn.

This result confirms that there is a problem in the HT93 theory concerning Iapetus.

\subsection{Comparison between observations and ephemerides of Saturn}

Here we try to find out the origin of the differences between the calculated positions of Saturn derived from DE403 and DE405. For this purpose we want to compare Saturn's observations with each of the theories. However Saturn cannot be directly observed with the 

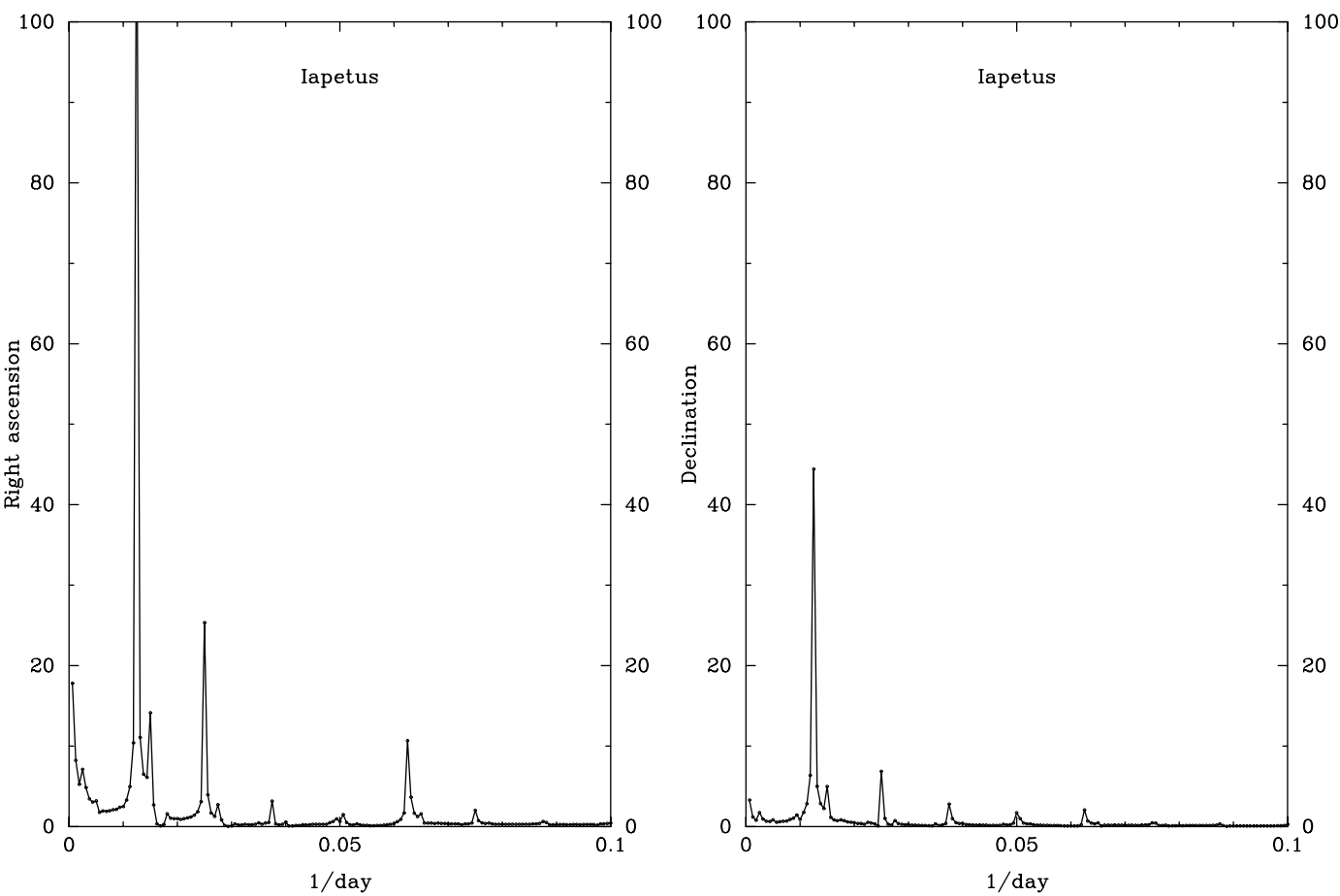

Fig. 6. Power spectrum of the difference between HT93 and VD95 theories.
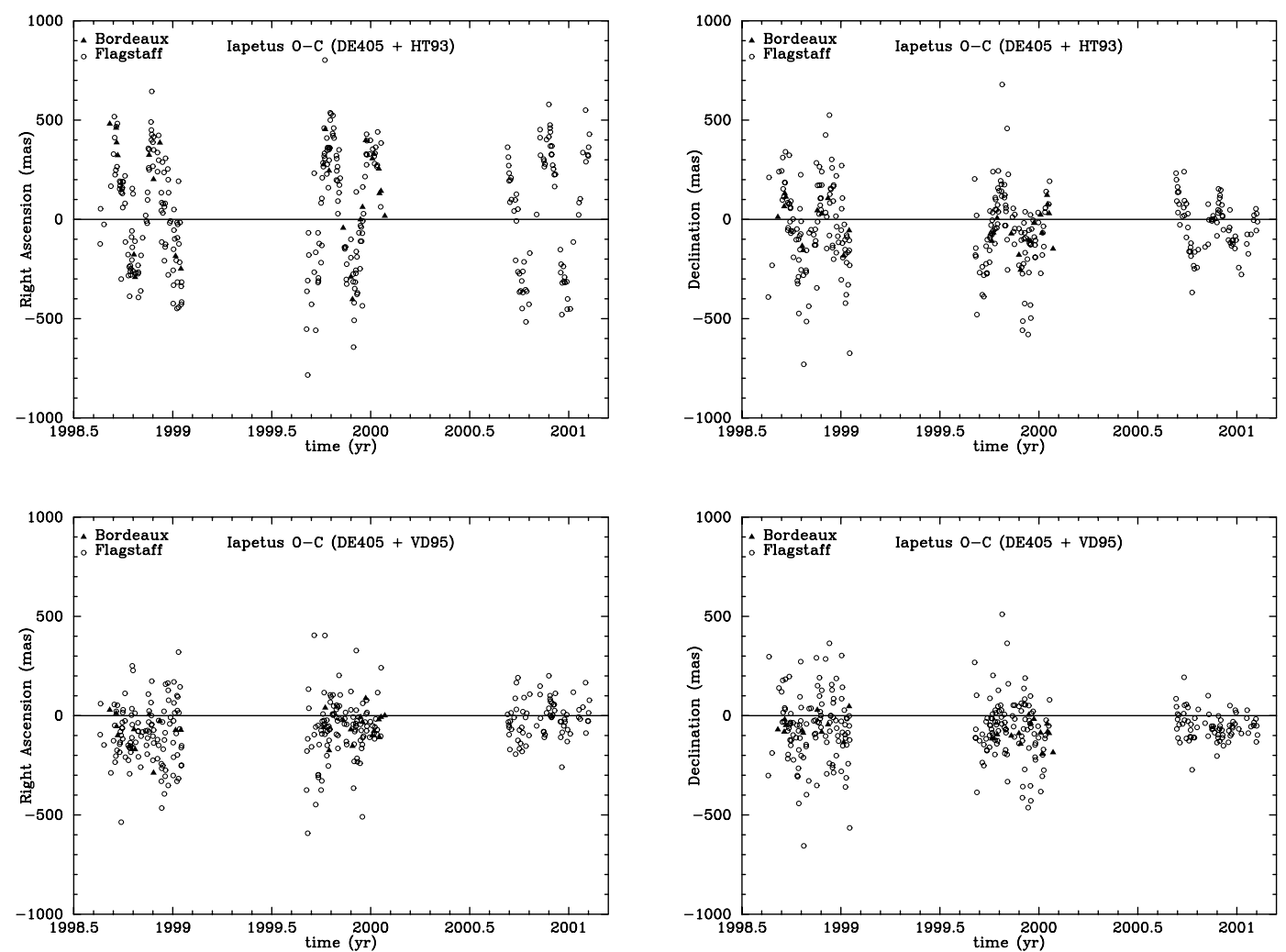

Fig. 7. Comparison of meridian observations of Iapetus with calculated positions $\left(\mathrm{C}_{\mathrm{DE} 405+\mathrm{HT} 93}\right)$ and calculated positions $\left(\mathrm{C}_{\mathrm{DE}} 405+\mathrm{VD} 95\right)$.

CCD meridian instruments because of its brightness. Thus we will compare the observed position of its satellites with their associated calculated positions. The calculated positions $=$ the vector Observer-Saturn ( DE403 or DE405) + the vector Saturn-Satellite (VD95 or HT93).
Then the differences observed between the observed and calculated positions of the satellite are attributed to an error in Saturn's calculated position since we assume that there is no systematic detectable error in the SaturnSatellite vector. 

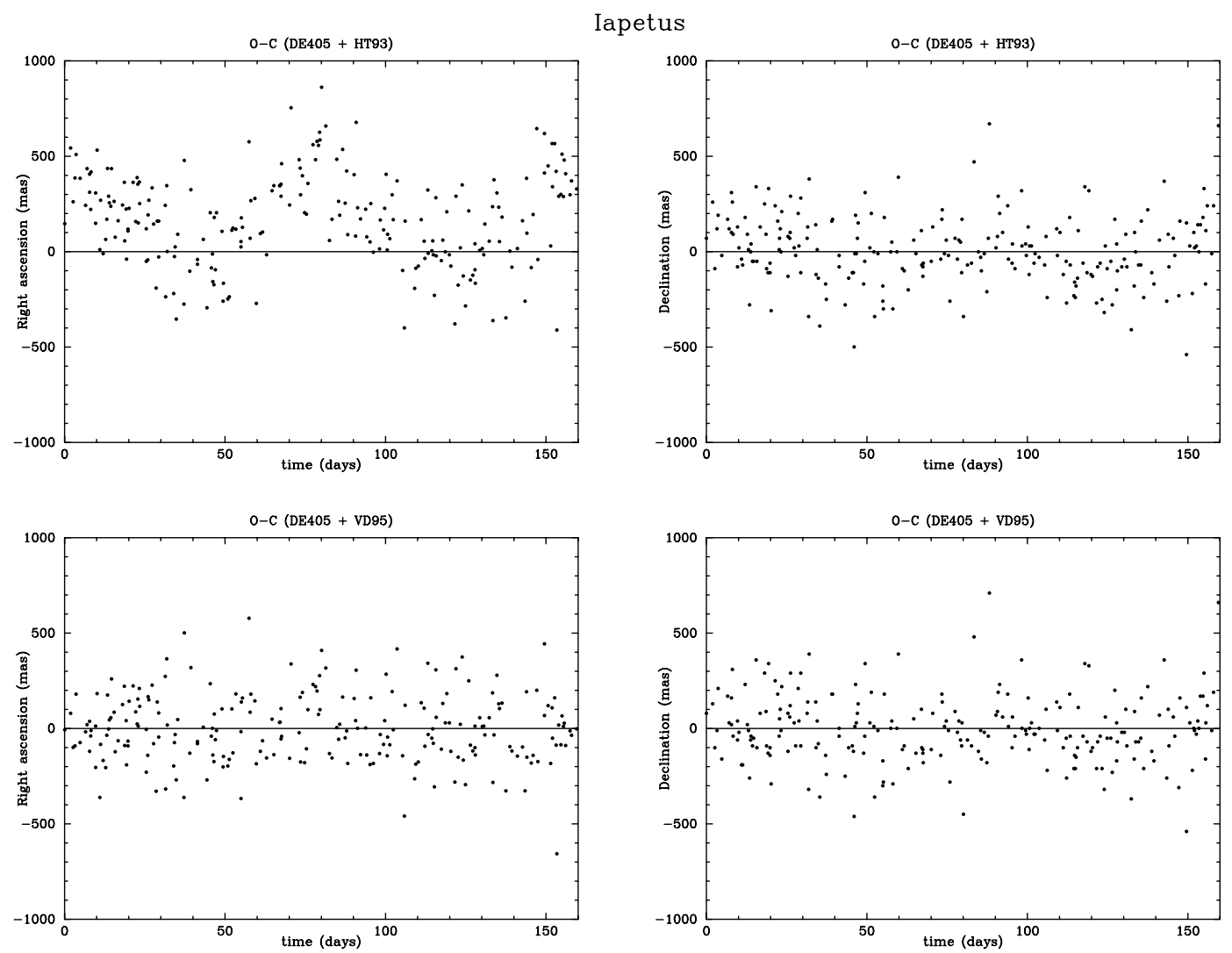

Fig. 8. Comparison of meridian observations of Iapetus with calculated positions $\left(\mathrm{C}_{\mathrm{DE} 403+\mathrm{HT} 93}\right)$ and calculated positions $\left(\mathrm{C}_{\mathrm{DE} 405+\mathrm{VD} 95}\right)$ versus time modulo 80 days. The presented observations were provided by the Carlsberg Automatic Meridian Circle and the Photoelectric Meridian Circle of Bordeaux between 1985 and 1997.
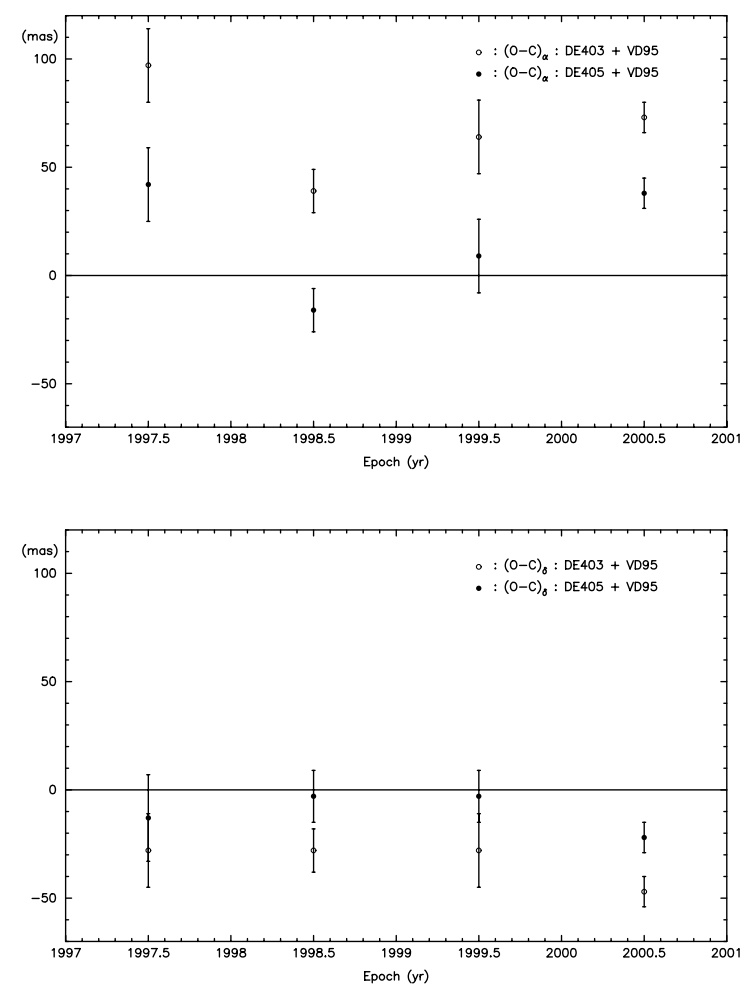

Fig. 9. $\overline{\mathrm{O}-\mathrm{C}}_{\mathrm{DE} 403+\mathrm{VD} 95}, \overline{\mathrm{O}-\mathrm{C}}_{\mathrm{DE} 405+\mathrm{VD} 95}$ and their associated rms (mas) for Saturn (derived from Rhea, Titan, Dione and Thetis observations).
We saw in the previous Sect. 5.3 that theories for satellites present differences less than 50 mas in geocentric positions except for Mimas and Iapetus (which obviously will not be used here to derive Saturn's position). We therefore suppose that the use of several satellites will minimise effects on Saturn's position due to possible remaining errors in the theories of satellites.

We used meridian observations of Rhea, Titan, Dione and Thetis to test the DE405 ephemeris of Saturn as described above. We present in Fig. 9 for each opposition, the $\overline{\mathrm{O}-\mathrm{C}}_{\mathrm{DE} 405+\mathrm{VD} 95}$ and $\overline{\mathrm{O}-\mathrm{C}}_{\mathrm{DE} 403+\mathrm{VD} 95}$ and their associated rms for Saturn.

The calculation of the weighted means of the $\mathrm{O}-\mathrm{C}$ for DE403+VD95 shows a systematic offset of about 65 mas in right ascension and -38 mas in declination with the observations. For DE405 the mean $\mathrm{O}-\mathrm{C}$ is much smaller and confirms the improvement attained by this ephemeris for Saturn too.

\section{Conclusion}

The analysis of CCD meridian astrometric observations of outer planets and of their satellites allows testing of the ephemerides of the planets and satellites.

The discrepancies between observed positions of Pluto and calculated ones based on DE403 are large. The DE405 ephemeris better represents the observations 
but some slight systematic effects remain, mainly in right ascension. In declination the differences are more chaotic and cannot easily be attributed to observations or ephemeris. The slight systematic offsets between observations and the DE405 ephemeris suggest that it is important to continue observation of Pluto.

In the case of Iapetus, we could discriminate between HT93 and VD95. Some periodic terms remain in the HT93 which have to be removed as suggested by Morrison \& Evans (1998). Periodic effects are absent in the VD95 theory which therefore should be used in the case of Iapetus. In this work, we also demonstrated that it was possible to use the observations of selected satellites of Saturn to show that the ephemerides DE403 of Saturn have to be improved and that DE405 well represents the observations.

For Uranus and Neptune, discrepancies between DE403 and DE405 are small at the present time (less than 50 mas) but in right ascension they will increase with time. It will be possible in the coming years to analyse the origin of these differences through CCD meridian observations.

Finally, the residuals obtained with observations performed with three different CCD meridian circles do not exhibit any systematic effects between the instruments. We conclude that this type of observation contributes significantly to the maintenance and the improvement of modern ephemerides of the outer planets.

Acknowledgements. The authors wish to thank the observers from Bordeaux and Valinhos for their regular participation. They also greatly thank J. Braine for his valuable suggestions on the text. They also wish to thank the following organisations for their financial support (CNRS, CNPq, COFECUB, FAPESP, CAPES).

\section{References}

Harper, D., \& Taylor, D. B. 1993, A\&A, 268, 326

Helmer L., \& Morrison L. V. 1985, Vistas Astron., 28, 505

Morrison, L. V., \& Evans, D. W. 1998, A\&AS, 132381

Réquième, Y., \& Mazurier, J. M. 1986, IAU Symp., 109, Astrometric Techniques, 435

Standish, E. M. 1990, A\&A, 233, 272

Stone, R. C. 2000, AJ, 120, 2124

Viateau, B., Réquième, Y., Le Campion, J. F., et al. 1999, A\&A, 134, 173

Vienne, A., \& Duriez, L. 1995, A\&A, 297, 588 